\title{
Interactive comment on "Diversity and distribution of Nitrogen Fixation Genes in the Oxygen Minimum Zones of the World Oceans" by Amal Jayakumar and Bess B. Ward
}

\section{Amal Jayakumar and Bess B. Ward}

ajayakum@princeton.edu

Received and published: 8 May 2020

In this study, the authors have reported the diversity and phylogeny of putative diazotrophs in the three major OMZs of the Ocean, including ETNP, ETSP and Arabian Sea. By analysing the clone libraries of nifH gene fragments derived from DNA and RNA samples (787 sequences in total), the authors compared the putative diazotroph communities in surface, oxycline and oxygen depleted water of the three OMZs. Basically, my major concerns are the significance of the finding and validity of the approach in this study. It should be noted that the phylogenetic diversity of putative diazotrophs in 
et al., 2012 \& 2017; Loescher et al., 2014; Cheung et al., 2016). Therefore, I afraid that the current study does not provide significant amount of new knowledge to the field. For the approach, $787 \mathrm{nifH}$ sequences are definitely insufficient to reconstruct the diazotroph communities in different layers of the three OMZs. The authors can calculate the coverage indices to evaluate whether the sequencing depths are enough. With such limited dataset, I doubt if it is meaningful and convincing to compare the diversity and community composition of putative diazotrophs in different waters. The authors stated that most of the OTUs were not shared among the regions ( $L$ 297), while it could also be the result of limited sequencing depth. Given that this study is mainly about phylogenetic diversity, the authors should consider using high-throughput next generation sequencing of nifH amplicons to provide more convincing findings.

Response: Thank you very much for your detailed constructive comments. We are addressing the specific comments below. In previous studies, what has been reported, describes the distribution of phylotypes and rates of dinitrogen fixation in the ETNP and ETSP, no rates for nitrogen fixation were made for the Arabian Sea. In this study we are comparing the distribution of the phylotypes of nitrogen fixing microbes and their expression, amongst the three major OMZs and also between the oxic and anoxic depths and their biogeography. We agree that number of sequences obtained from some of the depths are low compared to what could be obtained using next generation sequencing on new samples (which are not available for the Arabian Sea). True, the rarefaction curves (Figure 2 and Figure 5) indicate that sampling did not approach saturation, and for Cluster I there were many singletons, indicating more unexplored diversity. Next generation sequencing of nifH amplicons would provide a better understanding of the distributions but that would be a future study in itself - here we are presenting the synthesis that is possible for all three locations with the currently available data.

In the revised manuscript, we have compared our results more broadly to the few other available data sets from similar locations, and that synthesis points to some useful

Printer-friendly version

Discussion paper
Interactive comment (1) 
conclusions, which we now include in the discussion - see new text starting at L267 and L349. All the previous studies except Cheung et al. (2016) were based on clone libraries and they all, including Cheung et al (2016) find (where the data are available) 1 ) the same basic metabolic types and 2) dominance by a few OTUS in each sample. We interpret this pattern to reflect the presence of diverse assemblages that essentially bloom episodically in response to organic matter input. This interpretation makes sense for non-cyanobacterial clades in both clusters. The consistent detection of nifH genes from e.g., sulfate reducers and methanotrophs motivates further investigation of those metabolisms in ODZs.

Thus we think the conclusions from this study are indeed robust, and hypothesize that this new synthesis would be corroborated by more in depth sequencing.

Specific comments: 1) L30: The authors should briefly talk about the previous studies about the putative diazotrophs in OMZs, including the works done by other teams.

Response: We have introduced the other relevant studies in the introduction and incorporate comparisons with those studies in the results and discussion.

2) L45: Please provide detailed information about the sampling locations and depths.

Response: Thank you, Referee\#1 also suggested this and we now have Inserted new table with the information

3) L89-111: Details of qPCR assay were listed in the methodology, while the relevant result was not mentioned at all.

Response: Removed this section from methodology

4) L123: Please specify the sequence number of each sample.

Printer-friendly version

Response: The accession number ranges are now provided.

5) L137: The diverse nifH phylotypes of 4 different clusters and their affiliated strains have already been discussed in the previous studies. Is there any the new finding worth

Discussion paper 
elaborating? How about the correlation between nifH phylotypes and environmental variables?

Response: The new discussion sections starting at L L267 and L349 point out the new interpretations and ideas that are made possible by this synthesis of data from multiple sites and in comparison with previous studies. This is also highlighted in the conclusions.

6) L281: How about the other stations? How many stations in total? It may be easier to follow if the authors show the diaoztroph community composition in each station clearly.

Response: A new table 1 is now included which lists out the stations, positions and number of sequences obtained from each station and depths.

Interactive comment on Biogeosciences Discuss., https://doi.org/10.5194/bg-2019-445, 2020. 


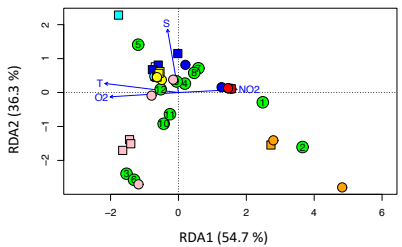

comment

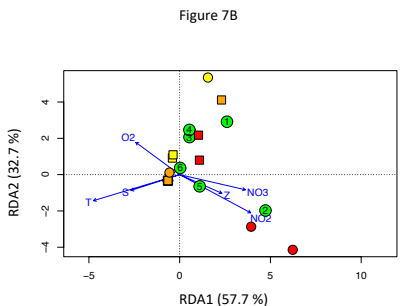

Printer-friendly version

Fig. 1. Figure 7. RDA plots for (A) Cluster I and (B) Clusters II, III, IV illustrating the relationships among OTUs (green circles) and sites. DNA = squares; $\mathrm{cDNA}=$ circles. Arabian Sea $=$ cyan (surface) a 